Case Reports

\title{
Transfusion Associated Peak in Hb HPLC Chromatogram - a Case Report
}

Sonal Jain, Jasmita Dass and Hara Prasad Pati

Department of Hematology, All India Institute of Medical Sciences, New Delhi

Correspondence to: Dr. Jasmita Dass, M.D. Department of Hematology, All India Institute of Medical Sciences, New Delhi. Tel: 9999347544, Fax: 01126588663. E-mail: drjasmita@ gmail.com

Competing interests: The authors have declared that no competing interests exist.

Published: January 21, 2012

Received: August 23, 2011

Accepted: November 11, 2011

Citation: Mediterr J Hematol Infect Dis 2012, 4(1): e2012006, DOI: 10.4084/MJHID.2012.006

This article is available from: http://www.mjhid.org/article/view/9068

This is an Open Access article distributed under the terms of the Creative Commons Attribution License (http://creativecommons.org/licenses/by/2.0), which permits unrestricted use, distribution, and reproduction in any medium, provided the original work is properly cited.

Abstract. High performance liquid chromatography (HPLC) and electrophoresis are commonly used to diagnose various hemoglobinopathies. However, insufficient information about the transfusion history can lead to unexpected and confusing results. We are reporting a case of Juvenile myelomonocytic leukemia (JMML) in which HbHPLC was done to quantify fetal hemoglobin (HbF). The chromatogram showed elevated $\mathrm{HbF}$ along with a peak in the $\mathrm{HbD}$ window. A transfusion acquired peak was suspected based on the unexpectedly low percentage of HbD and was subsequently confirmed using parental HbHPLC.

Introduction. It is well known that incomplete history on test request forms sent to laboratories and inappropriate patient samples can lead to wrong diagnosis and hazardous consequences. Hemoglobin electrophoresis and High Performance Liquid Chromatography (HPLC) are routinely done to diagnose and classify hemoglobinopathies. Acquired and inherited conditions in which abnormal HPLC result can be seen include high fetal hemoglobin in Juvenile Myelomonocytic Leukemia (JMML), Diamond Blackfan Anemia (DBA) and Fanconi Anemia. ${ }^{1}$ Blood transfusion from donors with hemoglobinopathies which are clinically silent (e.g $\mathrm{HbE}, \mathrm{HbD}, \mathrm{HbS}$ ) may lead to abnormal peaks or altered percentages of abnormal hemoglobins.

Case. A 7 year old female presented to the outpatient department with complaints of hepatosplenomegaly and lymphadenopathy for 3 months. Hemogram showed a hemoglobin of $9.5 \mathrm{gm} / \mathrm{dl}$, total leukocyte count of $57.24 \times 10^{9} / \mathrm{L}$ and platelet count of $16 \times 10^{9} / \mathrm{L}$. Peripheral smear showed $35 \%$ monocytes with an absolute monocyte count of $20 \times 10^{9} / \mathrm{L}$. Diagnosis of Juvenile myelomonocytic leukemia was suggested and $\mathrm{Hb}$ HPLC was advised to look for increased fetal haemoglobin. High Performance Liquid Chromatography (HPLC) was done using BioRad Variant II instrument with beta thalassemia short program. Hb HPLC chromatogram showed a raised $\mathrm{HbF}(11 \%)$ with $\mathrm{HbA}_{0} 69.2 \%, \mathrm{HbA}_{2} 1.8 \%$ and a peak in D-window of $10.2 \%$ with a retention time of 4.06 minutes (Figure 1). The expected percentage of $\mathrm{HbD}$ in heterozygotes is $\sim 40 \%$ but in our case it was significantly low. Hence, a possibility of transfusion acquired $\mathrm{HbD}$ was considered and $\mathrm{Hb}$ HPLC was done in both the parents. Both mother and father showed normal $\mathrm{Hb}$ HPLC (Figure $\mathbf{2 A}$ and 2B). The transfusion history was taken and it was discovered that the patient had received 3 units of PRBC one week prior to HPLC. Thus a final diagnosis of transfusion 


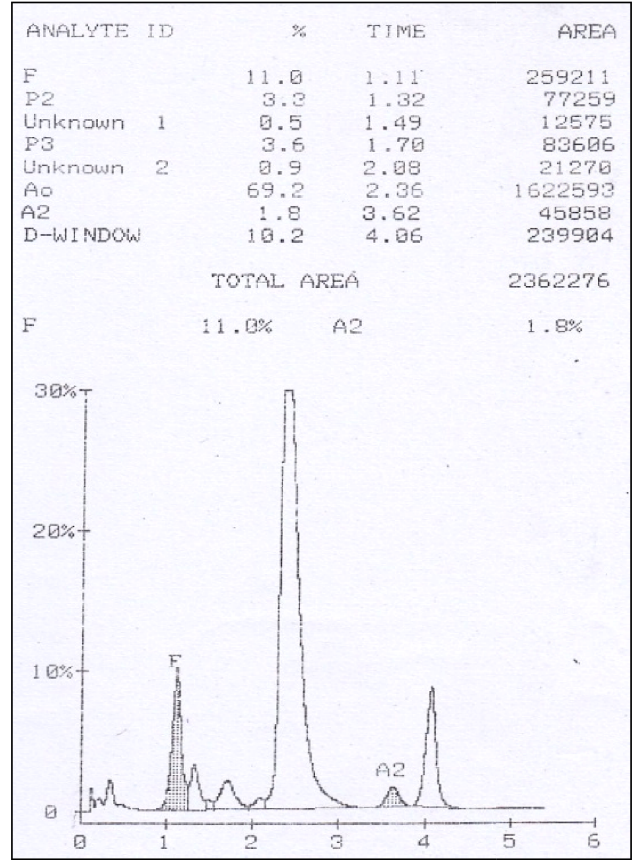

Figure 1. Hb HPLC of patient showing high $\mathrm{HbF}$ along with a peak in $\mathrm{D}$ window ( $\mathrm{RT}-4.06$ minutes). hemoglobin peaks following blood transfusions. ${ }^{2-5}$ In these reports, the abnormal hemoglobins account for $0.8-14 \%$ of the total hemoglobin. ${ }^{4}$ The largest series by Kozarski et al reported 52 occurrences of transfusion associated peaks in 32 recipients. The possibility of transfusion associated peaks was suspected on the basis of low concentrations, transient nature or in one case, with presence of three different peaks. ${ }^{4}$

The incidental finding of such abnormal peaks may cause diagnostic and therapeutic dilemmas even to the most experienced, particularly so in patients with suspected hemoglobinopathies who have received multiple blood transfusions. To avoid this rare problem some authors have suggested screening of blood donors for hemoglobinopathies. ${ }^{2}$ In this case, we want to highlight that a high index of suspicion along with careful clinical evaluation, family history and required investigations in parents and siblings, transfusion history and evaluation of unexplainable $\mathrm{Hb}$ percentages are some key features which can be helpful in correctly diagnosing this rare possibility.

associated peak in $\mathrm{D}$ window was made.

There are only a few reports of abnormal

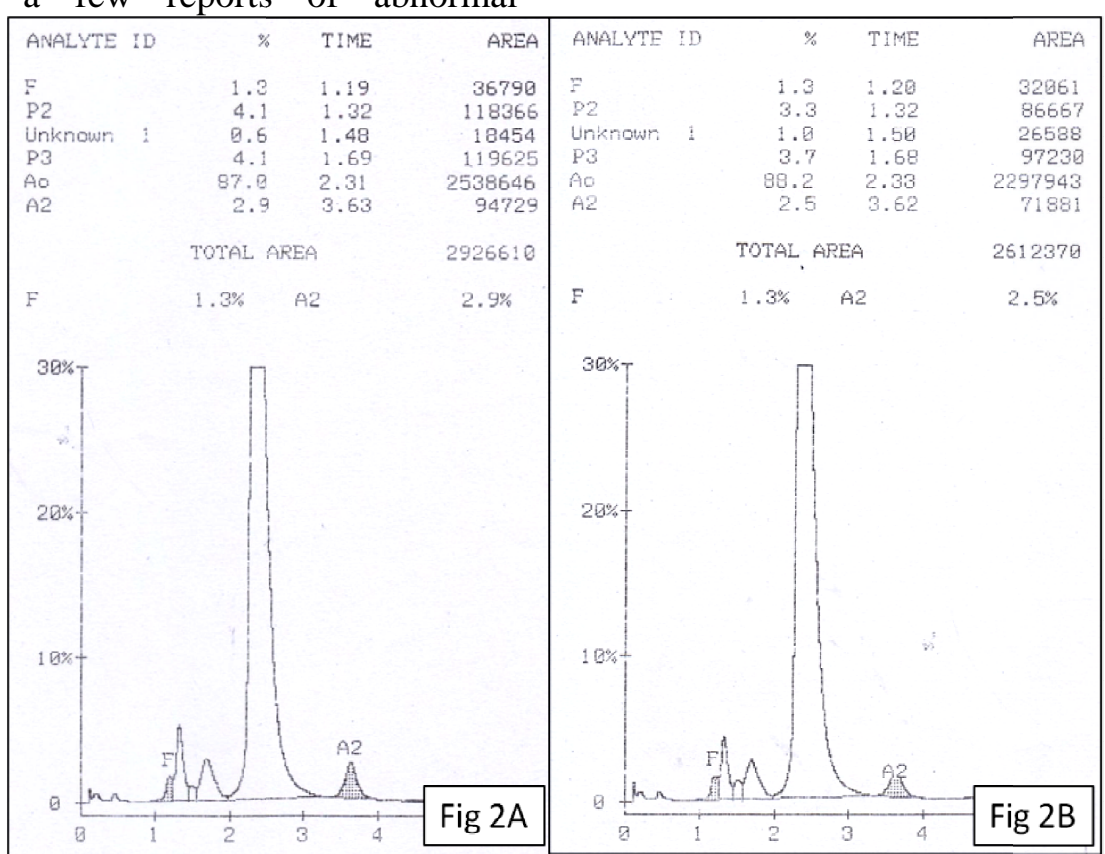

Figure 2. Hb HPLC of father (2A) and mother (2B) showing normal chromatogram.

\section{References:}

1. Teo JT, Klaassen R, Fernandez CV, Yanofsky R, Wu J, Champagne $\mathbf{J}$ et al. Clinical and genetic analysis of unclassifiable inherited bone marrow failure syndromes. Pediatrics 2008;122;e139-e148. http://dx.doi.org/10.1542/peds.2007-3415 PMid:18595958

2. Lippi G, Mercandanti M, Alberta C, Franchini M. An unusual case of a spurious, transfusion-acquired haemoglobin S. Blood Transfus 2010;8:199-202. PMid:20671882 PMCid:2906190

3. Suarez AA, Polski JM, Grossann BJ, Johnston MF M. Blood Transfusion-Acquired Hemoglobin C: A Case Report and Review of the Literature. Arch Pathol Lab Med. 1999;123:642-643. PMid:10388925

4. Kozarski TB, Howanitz PJ, Howanitz JH, Lilic N, Chauhan YS. Blood Transfusions Leading to Apparent Hemoglobin C, S, and OArab Hemoglobinopathies Arch Pathol Lab Med. 2006;130:18301833. PMid: 17149958

5. Wong P, Tapprom A, Jermnim N, Charoenporn, Kanthiyawong S. A blood transfusion leading to misdiagnosis of beta-thalassaemia carrier status. Blood Transfus 2010; 8:69-70. PMid:20104282 PMCid:2809515 